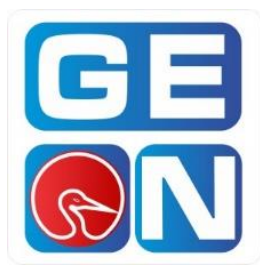

Revista GEON (Gestión, Organizaciones y Negocios.) ISSN: 2346-3910 en línea

revistageon@unillanos.edu.co

Universidad de los Llanos

Colombia

Soto Aguilar, Adriana Patricia.

Crisis en la política socio-comercial presidencialista norteamericana y sus efectos para la economía mexicana

Revista GEON, Vol. 4, No. 2, 2017

Pág. 116-124

Disponible en: $\underline{\text { https://doi.org/10.22579/23463910.29 }}$

Esta publicación se encuentra bajo licencia: Creative Commons

ReconocimientoNoComercialSinObraDerivada 4.0 Internacional

@) $\odot \Theta \Theta$

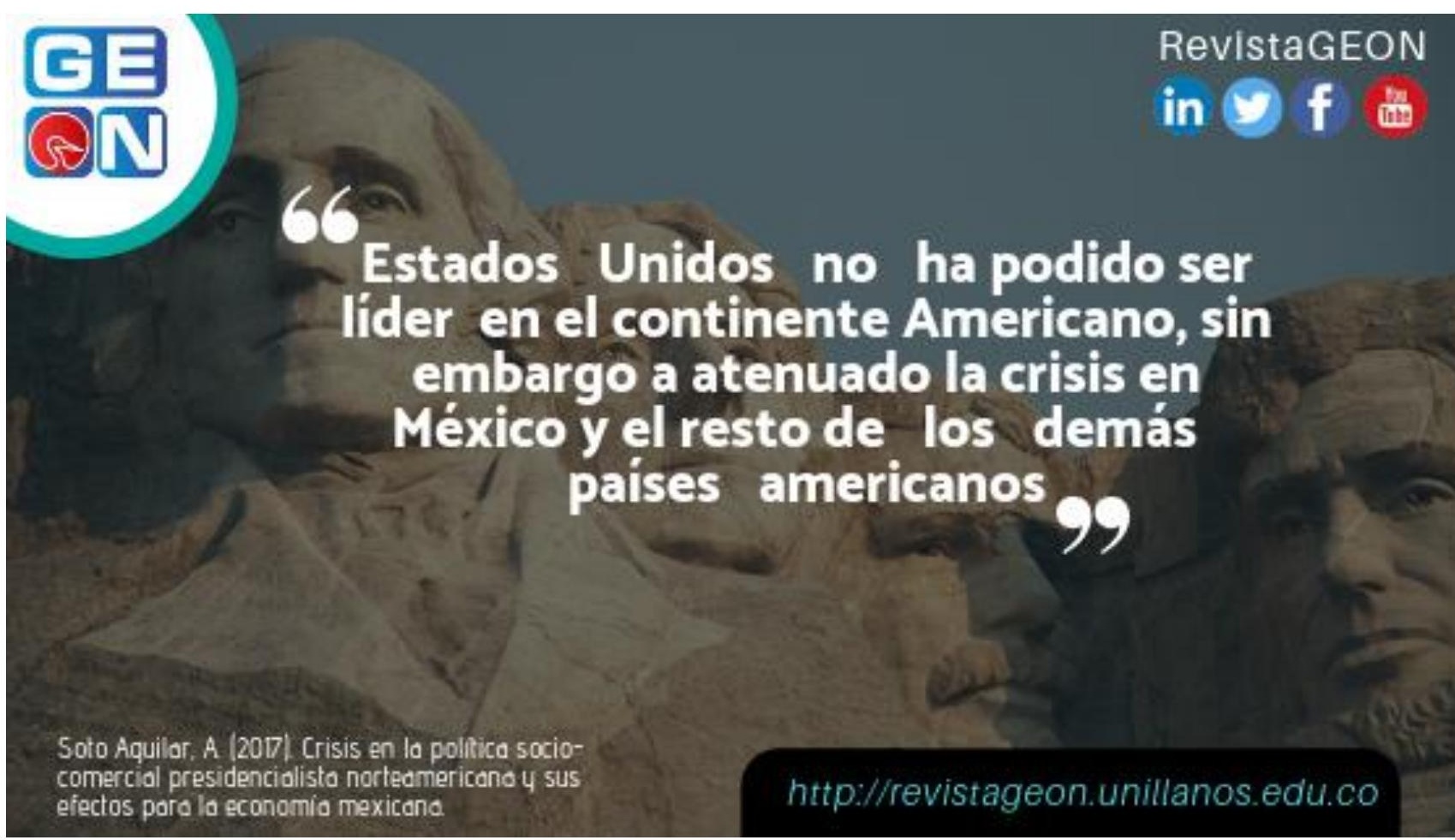




\section{Crisis en la política socio-comercial presidencialista norteamericana y sus efectos para la economía mexicana por Adriana Patricia Soto Aguilar}

\section{Crisis in the US presidential social-commercial policy and its effects on the Mexican economy by Adriana Patricia Soto Aguilar}

Adriana Patricia Soto Aguilar

Magister en Economía

Benemérita Universidad Autónoma de Puebla BUAP

adrianasotonegocios@gmail.com
Artículo recibido 2016/08/30

aceptado 2016/11/24

Escrito como parte de ponencia

Oral presentada en el IV Congreso

Internacional de Investigación

en Ciencias Económicas de la

Universidad de los Llanos

\section{Resumen}

Durante los últimos 10 años se ha observado un incremento de las contradicciones en la política económica comercial de la presencia de los Estados Unidos hacia México teniendo como resultado una inestabilidad en la relación comercial entre ambos países, hoy en día llama la atención las propuestas de los dos candidatos presidenciales a la Casa Blanca especialmente la política comercial y un escaso seguimiento por parte del Gobierno Federal Mexicano. El trabajo presentado pone énfasis en las propuestas comerciales de Donald Trump y en segunda instancia en los intereses ocultos de Hillary Clinton.

El reto de análisis es mayor dadas las crisis recurrentes de la Economía Norteamericana en materia inmobiliaria y crisis financiera especialmente el incremento de su deuda interna y cartera vencida, así como el incremento de las remesas hacia nuestro país. Se pretende Identificar las contradicciones de la política económicacomercial presidencialista norteamericana y sus efectos para México en torno a la crisis económica y financiera de ambos países, objetivo concluido, bajo la línea de investigación Crisis económica y financiera Internacional, como resultado relevante la política socio-comercial de Estados Unidos ha agudizado las contradicciones de las crisis recurrentes en Estados Unidos y en los Estados Unidos Mexicanos, generando nuevos procesos de acumulación de capital, y como conclusión apremiante se presenta en que la política socioeconómica del gobierno norteamericano y sus presidenciales aumenta los factores de crisis económica y financiera.

JEL A:13, D:60, F:18, N:35

Palabras clave: política económica-comercial, deuda interna, crisis financiera

\section{Abstract}

During the last 10 years there has been an increase in the contradictions in the commercial economic policy of the presence of the United States towards México, resulting in an instability in the commercial relationship between both countries. 
Nowadays, the proposals of the two presidential candidates to the White House especially the commercial policy and a little monitoring by the Mexican Federal Government. The work presented emphasizes the commercial proposals of Donald Trump and, secondly, the hidden interests of Hillary Clinton.

The challenge of analysis is greater given the recurrent crises of the North American Economy in real estate and financial crisis especially the increase of its internal debt and past due portfolio as well as the increase of remittances to our country. The aim is to identify the contradictions of the North American presidential economiccommercial policy and its effects on México regarding the economic and financial crisis of both countries, a goal that has been concluded, under the research line of the International Economic and Financial Crisis, as a relevant result -Commercial of the United States has sharpened the contradictions of recurring crises in the United States and the United States, generating new processes of capital accumulation, and as a pressing conclusion is presented that the socioeconomic policy of the US government and its presidential increases the factors of economic and financial crisis.

JEL A: 13, D: 60, F: 18, N: 35

Keywords: economic-commercial policy, internal debt, financial crisis

\section{Introducción}

La política exterior de Estados Unidos debe tener presente que para su país vecino enfrenta una frontera entre México y Estados Unidos tiene una extensión de unos 3 mil 200 kilómetros, siendo una de las más dinámicas del mundo. El 70 por ciento del comercio entre México y Estados Unidos, que asciende a unos 450 mil millones de dólares, se realiza con transporte de carga, mientras que alrededor de un millón de personas cruza diariamente en toda la frontera de México y Estados Unidos por cuestiones de trabajo, familiares, comercio, turismo o estudio.

El presente documento recapitula la política exterior de los Estados Unidos de Norteamérica con la República Mexicana en el contexto de la política migratoria, política laboral, política salarial, de inversión, así como las remesas que nuestros connacionales aportan a ambas economías, se analiza el doble discurso oficialista de los candidatos a la presidencia del gobierno norteamericano y las tendencias de los últimos gobiernos y sus políticas hacia México.
Cabe señalar que el estudio es de corte correlacional y se enfoca a las teorías imperialistas de John Atkinson Hobson, John Stuart Mill y Hegel entre otros más.

\section{Contexto teórico}

En las últimas décadas dentro de la relación México Estados Unidos se impone una política neoliberal raíz de las Cartas de Intención derivado de la Deuda externa impuesta por el FMI, Fondo Monetario Internacional, sin embargo una doble política norteamericana radica en imponer el neoliberalismo en México sin soberanía política, imponiendo mecanismos al capital trasnacional con privilegios monopolistas a costa del capital nacional y del cooperativismo incluso de comunidades productivas de carácter nacional, lo cual ha traído como consecuencia una disminución del ritmo de desarrollo en México pronosticado en $2.4 \%$ menor al $3 \%$ pronosticado (Forbes 2016), vinculada a la caída de precios, disminución de la tasa de ganancia y el incremento de la dependencia económica. 
Al final de cuentas sea la política de Reagan, Obama, Hillary Clinton o Donald Trump, lo que hay de fondo es una política imperialista, John Atkinson Hobson (185819940) destaca el liberalismo dentro del imperialismo atractivo para los trabajadores, situación nada ajena al incremento de los migrantes no solo mexicanos sino una tendencia mundial como lo vine Europa con los Africanos, contrariamente Estados unidos es el pastel atractivo de todo el mundo la mano de obra migrante no solo de México, hablemos de centroamericanos, latinoamericanos, cubanos, asiáticos, hindúes, y que decir de los Africanos asilados políticos en México que apelan no ser deportados y poder ingresar al territorio norteamericano, la pregunta es el problema para el imperialismo gringo y su política neoliberal son los mexicanos o los migrantes del mundo no hablo de los pobres marginados o indígenas incluyo a la clase media mundial y nuevos empresarios internacionales hábiles de estar en territorio gringo, de hecho en ningún momento se contradice a Hobson se hecho su idea se afirma, "la expansión era la búsqueda de oportunidades lucrativas" esta idea se observa desde la perspectiva de los círculos financieros en aras de un expansionismo derivado de la saturación del mercado interno, sin embargo esto es solo un análisis macroeconómico, si retomamos el eje microeconómico los migrantes estarían en el mismo derecho de ampliar sus ingresos cuando el mercado interno este contraído y que decir de la exclusión del tema de la mano de obra mexicana dentro del TLCAN, Tratado de libre Comercio con América del Norte sin dejar de lado el sub consumo derivado del bajo nivel de vida de la clase obrera, tal sub consumo ha incrementado los índices de crédito y deuda interna solo en México incluyo a Estados Unidos nada lejano con España, Grecia y parte de Europa la pregunta radica el llegar un empresario a la presidencia norteamericana agudiza las políticas económicas en favor de concentrar las prácticas monopólicas. Sin embargo se deja de lado el incremento del comercio armamentista con los países subdesarrollados, idea totalmente contraria a Jhosep Shumpeter quien plantea que dentro de las tendencias del capitalismo esta del desarrollo a través del comercio "El capitalismo es, por su propia naturaleza, antiimperialista" más bien Shumpeter se olvida que en el imperialismo existen contradicciones que destruyen al propio imperio, el propio mercado y su competencia asiática están disminuyendo la presencia norteamericana dentro del mercado mundial, a excepción de la economía de innovación que lideré Estados Unidos reafirmando la postura de John Stuart Mill en torno a que el imperialismo sortea el estancamiento por temporadas de ahí la necesidad de que la mano de obra mexicana y mundial ayuden a disminuir los costos de la mano de obra norte americana establecida legalmente, por ende la postura de Hegel en torno a la polarización social entre ricos y pobres hace la sociedad busque oportunidades en otros mercados laborales en tierras diferentes a las de nacimiento.

Complementando el análisis esta Hilferding Rudolf ministro de Finanzas durante el gobierno de Herman Muller indica que el capital financiero no busca la libertad necesita el sometimiento individual algo no lejano a la postura de Donal Trump, sigamos con la propuesta de Rodulf Hilferding, aborrece la competencia, nada lejano a anular el Tratado de libre comercio e imponer un mercado salvaje es decir requiere un estado políticamente poderoso que pueda imponer su propia política comercial y financiera a sus socios o países dependiente es decir afianzar sus intereses imperialistas contrariamente a los de sus socios comerciales y así ejercer presión política mundial en busca de condiciones comerciales favorables a sus intereses particulares para poder intervenir aún más en cualquier parte del mundo es decir continuar con su política expansionista.

\section{Materiales y métodos}

El presente trabajo representa meses de investigación documental. El tipo de estudio de esta investigación es exploratorio, descriptivo y correlacional: El estudio exploratorio nos sirvió para aumentar el grado de familiaridad con el fenómeno relativamente desconocido sobre la política comercial, obtener información sobre la posibilidad de llevar a cabo la investigación más 
completa sobre un contexto particular de la vida real, como es el caso de las oportunidades comerciales que ofrece la relación económica entre ambos países además de investigar problemas del comportamiento humano que consideren cruciales dentro del área de la política económica, identificar conceptos o variables promisorias, establecer prioridades para investigaciones posteriores y sugerir nuevas líneas de investigación (postulados) verificables (Dankhe, 1986).

En cuanto al método descriptivo, se aplicó al detectar y definir ciertas variables $y$ generalizaciones sobre la promoción comercial. En este caso la investigación se inició como descriptiva o correlacional, pues se descubrieron ciertas variables sobre las cuales se fundamenta el estudio. Asimismo, fue posible adicionar variables a medir. Los estudios descriptivos buscaron especificar, las propiedades importantes de personas (empresarios), comunidades (mexicanas en Estados Unidos) o cualquier otro fenómeno (comercial), que sea sometido a análisis, por lo que el estudio es correlacional (Sampieri, 2013) ya que los antecedentes nos proporcionaron generalizaciones para vincular variables e hipótesis sobre las cuales se trabajó.

\section{Resultados}

Bloqueo de remesas de inmigrantes. Independientemente de quien quede en la presidencia de los Estados Unidos de Norteamérica existe una fuga de capitales de la economía norteamericana, tan solo para México las remesas de los mexicanos en el exterior suman de enero a julio de 2016, 15,390 millones de dólares, con un incremento de $7.5 \%$ respecto al mismo periodo del año pasado. Observar cuadro No. 1, Sin embargo cuando se genera una disminución de las remesas (1.4\% agosto 2016) en automático se incrementa el ahorro de los migrantes mexicanos en el extranjero o bien la devaluación acelera el poder adquisitivo en México, hace nueve días se encontraba en 18.27 pesos por dólar el costo financiero es del $2.7 \%$ del PIB lo que provoca también contraer la demanda de productos norteamericanos entre
México y Estados Unidos, la cual representa la cuarta economía del mundo esta se calcula en un millón de dólares por minuto y la mayoría pasa por vía terrestre de hecho son más de catorce millones de consumidores en 10 estados de ambos países quienes realizan un intercambio comercial formal y legal dentro de la franja fronteriza es decir independientemente de los gobiernos y sus presidencialistas se tiene una vida propia del comercio mundial principalmente Tijuana, Texas, Los Ángeles, NY, Arizona, Baja California, Sonora, Chihuahua y Nuevo México, ventas cercanas a 94 mil 800 dólares por región, en pocas palabras solo se perjudica a México y queda intacta la economía norteamericana????, lo cierto que la reducción presupuestal del gasto gubernamental se da entre ambas naciones, en México en lo que va del año se ha generado dos recortes presupuestales tan solo del 2012 al 2015 solo aumento 1.9 puntos del PIB mexicano, sectores desfavorecidos son el área de la salud, los programas educativos, Petróleos Mexicanos.

\section{Cuadro No. 1 Remesas en México}

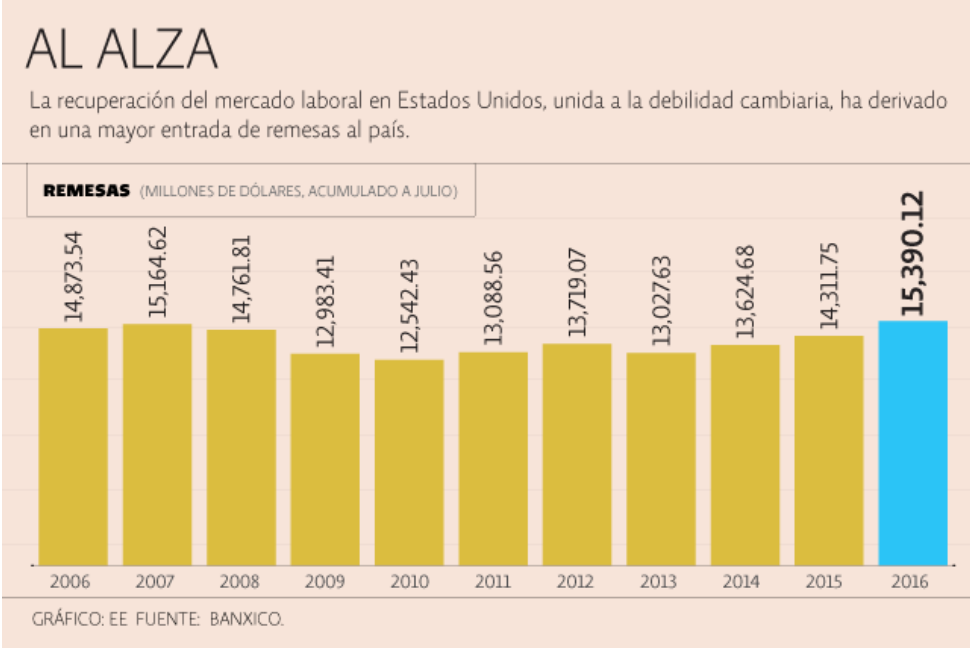

Mientras que para Estados Unidos el presidente Barack Obama y el grupo republicano han fracasado su déficit presupuestal sumo en el 2013 un billón de dólares (Bautista 2016). Por otro lado no se observa en los discursos de los candidatos presidenciales norteamericanos la importancia comercial entre ambas naciones, Incluso las cifras del Departamento de Comercio de los Estados unidos de América señala que en el caso de los Estados Fronterizos como California, Arizona, Nuevo México y Texas 
dependen seis millones de empleos solo en Estados Unidos, ya que Estados Unidos es el primer país extranjero por sus inversiones en la República Mexicana en marzo de 2013 la inversión de norteamericanos en nuestro país fue de 156, 000 millones de dólares, cifra independiente del turismo el cual ha disminuido por efectos de inseguridad pero esto le sucede a ambos países.

Políticas Migratorias.-Contrariamente las políticas migratorias de los norteamericanos es la militarización de las frontera México-Estados Unidos lo que ha llevado a un sin número de violaciones de derechos humanos y roces diplomáticos incluso el alto comisionado de Derechos Humanos de la ONU (Organización de Naciones Unidas), Zeid Ra'ad Al Hussein, (2005) además de señalar que los Estados Unidos conserva la mayor infraestructura de detención de inmigrantes de todo el mundo, con un costo de dos millones de dólares anuales, la problemática se agudiza debido a que dentro de las detecciones también se genera la violación de derechos humanos de los infantes en los propios centros de detención se señalan reportes de violencia y abusos contra menores en el mejor de los casos o bien secuestros, asesinatos, desapariciones, tráfico de órganos, violencia sexual, explotación infantil o abuso laboral a esto se le agrega el registro de datos oficiales solo se queda el reporte de los menores o de sus padres y mentores, violentando la Ley de Migración, atentando en contra su dignidad humana, generando acciones de inconstitucionalidad ya que se genera un déficit en la información al comparar los datos de la Oficina de Aduanas y Protección Fronteriza de Estados Unidos (US Customs and Border Protection, CBP) con los datos del Instituto Nacional de Migración (INM), durante los primeros siete meses del año fiscal 2015, tan solo la Patrulla Fronteriza (BP, por su sigla en inglés) capturó a 9,802 menores no acompañados procedentes países, centroamericanos mientras que en el periodo comprendido entre octubre del 2014 y abril del 2015, CBP detuvo a 70,448 migrantes "no mexicanos" en la frontera sur de Estados Unidos, durante este mismo período, mientras que las autoridades mexicanas detuvieron a 92,889 centroamericanos en México derivado de la pauperización que vive el continente lo que arroja enormes cantidades de ciudadanos que siguen huyendo de sus países pero la mayoría de ellos están siendo capturados en México, en lugar de Estados Unidos, es decir el país le sirve a la política migratoria de su socio comercial, que hoy en día se convierte en una crisis humanitaria que amenaza a las libertades civiles, reitero los fenómenos de migración no son un problema exclusivo de México, las crisis financieras, de mercado y de desempleo es un reflejo de la poca visón neoliberal ya que en México se practica el ochenta veinte es decir el ochenta por ciento de los mexicanos soporta lo básico para vivir mientras que la riqueza solo se concentra en el veinte por ciento restante, nuestros connacionales buscan en la migración hacia los Estados Unidos como una válvula de escape a la crisis económica que vive el país y a la ola de violencia producto del narcotráfico la entrada a los Estados Unidos no es una opción para los mexicanos es una necesidad con carácter histórico, sin embargo la entrada al país vecino no es exclusiva del mexicano están los propios asiáticos, centroamericanos, etc, etc y que decir de los africanos en territorio mexicano que viajaron en avión hasta Brasil, y de allí emprendieron un trayecto por autobuses, botes y a veces caminando, entre Perú, Ecuador, Colombia, Centroamérica y el sur de México, en Tapachula, Chiapas, ciudad fronteriza con Guatemala, mientras consiguen dinero para viajar a Ciudad de México, y de allí a Estados Unidos:

"Salí de mi país por problemas políticos, estaba en un partido de oposición. Ahora estamos buscando un lugar donde podamos vivir mejor. Los jóvenes forman parte de una silenciosa oleada de migrantes africanos que desde hace varios meses llegan a México" Fahrid septiembre 2016, o bien de los cubanos con la Ley Pública 89-732 de 1966 conocida como 'Ley de Ajuste Cubano', la política llamada comúnmente 'pies secos, pies mojados' se ha constituido, en un estímulo al flujo desordenado, irregular e inseguro de ciudadanos que arriesgan sus vidas, medidas o políticas que someten a los inmigrantes a una serie de injusticias. Ya que son detenidos de manera 
rutinaria y arbitraria sin derecho a fianza, se les ha negado el acceso a algún abogado, son deportados sin apelación y castigados por infracciones no justificadas, o menores, lo cual no permite misericordia de un juez o de la corte norteamericana, incluso la política más radical dentro de los Estados Unidos señala que los inmigrantes deberían ser tratados como una clase de sospechosos criminales, lo cual incita al incremento de las actividades de los grupos como el $\mathrm{Ku}$ Klux Klan enfocados hacia migrantes, generando un clima de neo racismo.

A los políticos norteamericanos se les ha olvidado que más de 2.500 personas murieron en Estados Unidos el 11 de septiembre de 2001, entre ellos connacionales mexicanos ahora "criminales" y que, a poco tiempo del decimoquinto aniversario del atentado, el grupo terrorista y el líder red terrorista Al Qaeda, Ayman al Zahawiri, ha amenazado con repetir "mil veces" los atentados terroristas, (John Brennan 2016.). Al contrario, nuestro territorio mexicano se vuelve vulnerable ante estos atentados, factura que no pagara los presidencialistas a la Casa Blanca.

¿La pregunta en torno a esta problemática es saber si la migración es un problema entre México Estados Unidos o bien es una tendencia mundial? La tensión sobre la migración en nuestro continente ha orillado a un pronunciamiento extra territorial en Quito Ecuador. Cancilleres de nueve países de América Latina han pedido e al gobierno de Estados Unidos revisar la política migratoria de este país hacia migrantes, lo cual tiene efectos negativos en la región. La postura diplomática fue a través de una carta enviada al Secretario de Estado, John Kerry y entregada por la misión de Quito en Washington, ministros de Exteriores de Ecuador, Colombia, Costa Rica, El Salvador, Guatemala, México, Nicaragua, Panamá y Perú, han manifestado profunda preocupación por el impacto de las normativas migratorias estadounidenses con respecto a los ciudadanos del continente.

Política salarial.-Las leyes actuales sobre migración son perjudiciales principalmente entre ambas naciones; dada la aportación de la economía mexicana a los Estados Unidos y la mano de obra barata y sin ninguna prestación debería ser legalizada, sin embargo la política de migración tanto en Europa como en los Estados Unidos es ilógica, disfuncional e inefectiva contrariamente a lazos comerciales, políticos y de inversión con México, "La ley de inmigración de los Estados Unidos ha sido abrumada por la realidad económica. La demanda por mano de obra poco calificada continúa creciendo en los Estados Unidos mientras la oferta doméstica de trabajadores adecuados disminuye inexorablemente. La administración Bush y el Congreso deberían trabajar en conjunto para forjar una política limítrofe que se acomode a las necesidades y aspiraciones de la gente en ambos lados de la frontera.... el número de inmigrantes indocumentados en los Estados Unidos hoy en día está alrededor de ocho millones, El presidente Bush y los líderes de ambos partidos en el Congreso deberían retomar la tarea de transformar el sistema disfuncional de inmigración de Estados Unidos "Daniel Griswold 2016 ,

Los norteamericanos enfrentan una política laboral que no cualquier país tiene por ejemplo las sanciones a los patronos, incremento del financiamiento para armamento y personal de control fronterizo desde 1986, lo que es una carga financiera para ambas naciones genera además un comercio y una industria ilegal de contrabando, la migración va acompañada del fraude de documentos a cuotas de dinero y otras actividades criminales en ambos territorios; la política actual de migración ha contribuido a la muerte de cientos de inmigrantes en la frontera y los que logran internarse a territorio norteamericano trabajan en la sombra, en la detención, con miedo, trabajan silenciosamente en una economía subterránea sin el leyes o protección alguna mientras para los conservadores norteamericanos esto tiene un signo de dólares: incrementar en 3 mil agentes a la Patrulla Fronteriza y mil millones de dólares más para el muro transfronterizo.

Ahora bien si analizamos la política salarial no estamos tan ajenos de los estándares 
internacionales esta política migratoria tiene su beneficio para los trabajadores pues han disminuido los salarios en términos reales los norteamericanos ahorran en mano de obra cerca de $\$ 3.000$ millones al año, es decir la mano de obra barata y maltratada no es peso para los contribuyentes norteamericanos al contrario es una caja de ahorro en todos los niveles tanto empresarial como en los hogares domésticos norteamericanos, las visas temporales monitoreada puede ser una alternativa para la migración México-Norteamérica.

De hecho en el 2009, los connacionales apoyaron y cargaron también la crisis financiera de Estados Unidos la cual pudo evitarse dada la excesiva cartera del riesgo y la negligencia de los reguladores financieros Alan Greenspan 2011 (Ex presidente de la Reserva Federal) unido al incremento desmedido de las hipotecas e instrumentos de inversión -boom crediticio- los cuales se comercializaron en grandes volúmenes por los propios bancos de inversión norte americanos quienes fallaron en los niveles de calificación crediticia para reconocer los riesgos involucrados en las carteras de los portafolios de inversión, deuda hipotecaria y burbuja inmobiliaria.

Sin embargo el Gobierno norteamericano, candidato, señala que la crisis que vive Estados Unidos es provocada por los migrantes mexicanos, sin embargo nunca mencionan que el Banco de la Reserva Federal de nueva York bajo la tutela de Tim Geithner y Henry Paulson y las firmas de Wall Street pudieron haber tomado las medidas financieras contra los excesos de Citigroup y atenuara la incertidumbre y el pánico del mercado bursátil poco valen los informes de 576 páginas, entrevistas con 700 testigos y 19 días de audiencias si el pronunciamiento de candidatos a la presidencia es la culpa de la crisis en Estados Unidos son los migrantes mexicanos y que decir de los resultados de su política armamentista contra Irak e Irán, o de su socio comercial China quien ha entrado a la carrera armamentista, en un nuevo cambio geopolítico. Es de todos sabidos que si estados Unidos es una potencia económica también es una potencia militar de los cuales los inmigrantes mexicanos han formado parte de sus listas militares como parte del servicio social a la patria que no es la suya.

Hay que señalarlo la guerra del Golfo es una forma bélica de sacarle el petróleo a Medio Oriente sin hacer más sacrificios económicos y con ello imponer una política de presionar el precio del petróleo otra variable más que nos afecta a los mexicanos ante la política petrolera de nuestro socio comercial ya que para el Secretario de Energía de la Administración Carter el tema del petróleo es considerado como un tema de seguridad mundial ya que este asciende a 76 millones de barriles consumidos por día se estima que llegue a 120 millones para el 2020 de estos Estados Unidos consume el 26 por ciento no de la nada los últimos gobiernos norteamericanos tienen en sus filas relaciones estrechas con líderes petroleros, observar listado No. 1.

Estados Unidos es quien más necesita asegurar el acceso y el control de las reservas de petróleo incluidas las del territorio mexicano y en la mira el petróleo de origen venezolano. De las 20 principales empresas en el mundo petrolero 13 son estatales y sólo 7 son del sector privado.

Los rumores internacionales en torno a Angola y la intervención de los Estados Unidos en su política interna no se han hecho esperar se habla de un posible financiamiento de la guerrilla en Angola líder petrolero en el continente africano por parte de los norteamericanos o bien el Plan Colombia donde Estados Unidos estará controlando la región comprendidas por Ecuador, Venezuela, Colombia y Perú lo mismo que sucedió en Vietnam y el caso Mobil empresa norteamericana o el caso Chechenia y Europa para tratar de romper la presencia de Rusia en dicha zona, casos similares con la ex-Yugoslavia igualmente los conflictos en torno a los yacimientos en el Medio Oriente, el Caspio hasta el mediterráneo.

Sin dejar de lado Azerbaiján, Kazajstán, Turkmenistán y Uzbekistán juntos estos paises tienen 115 mil millones de barriles de reservas probadas de crudo y 11 trillones de $\mathrm{m} 3$ de gas, Rusia ha mantenido la hegemonía en el transporte de crudo en la región, la misma que quiere ser arrebatada por Estados Unidos 
Listado No. 1: Empresas Petroleras Nacionalidad Propiedad Producción (miles b/d)

\begin{tabular}{|c|c|c|}
\hline 1.Arabian American Oil Company & $\begin{array}{l}\text { (Aranco) Arabia Saudí Estatal } \\
7.915\end{array}$ & $\begin{array}{l}\text { 2.National Iranian Oil Co. (NIOC) } \\
\text { Irán Estatal } 3.660\end{array}$ \\
\hline 3.Petróleos Mexicanos (PEMEX) & 4.Petróleos de Venezuela SA & (PDVSA) Venezuela Estatal 3.252 \\
\hline México Estatal 3.460 & & \\
\hline 5.Irak National Oil Co. (INOC) Irak & 6.Exxon Mobil EUA Privada & 7.Royal Dutch / Shell Reino U./ \\
\hline Estatal 2.573 & 2.501 & Holanda Privada 2.279 \\
\hline 8.PetroChina R. P. China Estatal 2.096 & $\begin{array}{l}\text { 9.Nigerian National Petroleum } \\
\text { Corp Nigeria Estatal } 2.038\end{array}$ & $\begin{array}{l}\text { 10.Chevron Texaco EUA Privada } \\
1.962\end{array}$ \\
\hline $\begin{array}{l}\text { 11.Abu Dhabi National Oil Co. } \\
\text { Emiratos Arabes Estatal } 1.904\end{array}$ & $\begin{array}{l}\text { 12.British Petroleum-Amoco } \\
\text { (BP }\end{array}$ & Amoco) Reino Unido Privada 1.890 \\
\hline 13.Kuwait Petroleum Co. (KPC) & 14.OAO Lukoil Rusia Privada & 15.TotalFinaElf Francia/ Bélgica \\
\hline Kuwait Estatal 1.633 & 1.553 & Privada 1.433 \\
\hline 16.Libian National Oil Corp. (Libia & NOC) Libia Estatal 1.416 & $\begin{array}{l}\text { 17.Petróleo Brasileiro (Petrobras) } \\
\text { Brasil Estatal } 1.274\end{array}$ \\
\hline 18.Pertamina Indonesia Estatal 1.268 & $\begin{array}{l}\text { 19.OAO Yukos Rusia Privada } \\
992\end{array}$ & $\begin{array}{l}\text { 20.Petroleum Development Oman } \\
\text { Omán Estatal } 841\end{array}$ \\
\hline
\end{tabular}

Fuente: Informe especial de Oil \& Gas Journal del $1^{\circ}$ de octubre de 2013

La devaluación del peso mexicano.- cada vez que en México se genera una devaluación existe un fenómeno dual para ambos países, para México el proceso de las importaciones se encarece lo que hace menos atractivo la importación de artículos del extranjero a la larga obligara a los empresarios mexicanos y al propio gobierno a invertir en nuevas inversiones para atender la demanda interna, situación nada fuera de lo real ya que el consumo en México ha "disminuido" por no indicar que el incremento es relativo, observar cuadro No. 2 (INEGI, agosto 2016)

Cuadro No. 2 Índice de volumen físico 2008=100, 2016P

\begin{tabular}{|c|c|c|c|c|c|c|c|c|c|c|}
\hline Denominación & Ene & Feb & Mar & Abr & May & Jun & Jul & Ago & Sep & Oct \\
\hline Total & 119.2 & 115.2 & 118.1 & 115.5 & 118.8 & 120.3 & & & & \\
\hline Nacional & 121.5 & 116.3 & 119.1 & 116.5 & 120.2 & 121.5 & & & & \\
\hline Bienes & 120.2 & 110.8 & 113.7 & 112.3 & 117.2 & 118.9 & & & & \\
\hline Servicios & 122.8 & 121.9 & 124.5 & 120.6 & 123.2 & 124.2 & & & & \\
\hline Importado & 98.3 & 104.7 & 109.8 & 107.3 & 107.0 & 109.1 & & & & \\
\hline Bienes & 98.3 & 104.7 & 109.8 & 107.3 & 107.0 & 109.1 & & & & \\
\hline
\end{tabular}

PCifras preliminares

\section{Conclusiones}

Ambos países tienen un vínculo geográfico, histórico, económico y cultural (la cultura chicana) además de la familiar que ha permanecido impermeable entre ambas naciones. Los recortes presupuestales entre ambas naciones provocan un estancamiento y una desaceleración que perjudica a ambas naciones, crecimiento económico, contaminación, violación de derechos humanos, así como los problemas y desafíos que se enfrenta los gobiernos presidencialistas.

Para el caso de México el resultado de la política comercial en la últimas décadas arrojo un incremento sustancial de la presencia asiática, las importaciones son mayores a las exportaciones, hoy en día más de la mitad de las importaciones que realiza nuestro país provienen de nuestro país vecino: Estados Unidos, con un 50.2\% (INEGI 2012), de China un $14.7 \%$, Japón un $4.7 \%$ y por 
último del resto de los países 30.4\%,especificamente Canadá, Brasil, Alemania, Italia, Corea del Sur, Japón y Taiwán, en México y en todo el mundo se va a sentir el golpe económico de los recortes en el gasto público en los Estados Unidos quien en los últimos periodos ha incrementado sus tasas impositivas, los recortes presupuestales norteamericanos están estimados en más de 85 , 000 millones de dólares y una perdida estimada de empleos de hasta 750000 trabajadores conjuntamente con una reducción presupuestal de medio punto porcentual en el crecimiento del PIB norteamericano.

Contrariamente Estados Unidos no ha podido ser líder en el continente Americano, sin embargo a atenuado la crisis en México y el resto de los demás países americanos, quien han seguido fielmente la política de reducción de aranceles, apertura a la iniciativa privada extrajera, venta de para estatales, reducción de la burocracia, más apertura comercial, privatización de la educación y el resto de la actividad financiera, nula inversión estatal, control salarial, abuso en el crédito al consumo y castigo al ahorro, fuja de capitales entre otros más resultados. En síntesis, en opinión de Perry Anderson, la reorganización del mundo o, en otros términos, del orden mundial se ha realizado bajo el liderazgo estadounidense, con instituciones económicas, políticas y de seguridad que claramente benefician su predominio geoestratégico y económico.

\section{Referencias}

Alan

Greenspan

2011 ,

http://www.bbc.com/mundo/noticias/2011/01/110127 economia eeuu crisis evitable jrg.shtml

Alberto Nájar, BBC Mundo, Ciudad de México, marzo 2016

Díaz Bautista, Alejandro economista y catedrático del Colegio de la Frontera Norte (Colef), 2016
Dankhe, G. (1986) citado en Hernández, R., Fernández, C., Baptista P. (1998). Metodología de la Investigación. (segunda edición). México, D.F.: Mc Graw Hill.

Fahrid a BBC Mundo, Ciudad de México, septiembre, 2016

Giulietto Chiesa, analyste politique de réputation internationale. Il est membre du Club Valdaï, de la conférence Axis for Peace et est membre du Bureau exécutif du World Policial Forum., JPEG 21.9 KB Humberto Salgado / Agence IPI, http://www.voltairenet.org/article154346.html

Griswold Daniel, director asociado del Centro de Estudios de Política Comercial de Cato Institute http://www.elcato.org/la-politica-migratoria-de-eeuulimita-en-lo-absurdo

Zeid Ra'ad Al Hussein, http://eleconomista.com.mx/sociedad/2015/06/15/pol iticas-migratorias-mexico-como-las-eu, , Hernández Leopoldo

Hilferding Rudolf, El capital Financiero, 1910, Fondo de cultura Económica FCE

Hobson, John Atkinson, Estudio del imperialismo, 1902, recompilado Fondo de Cultura Economica, México.

INEGI, Instituto Nacional de Estadística Geografía e Informática, México agosto 2016

John Brennan, entrevista Al Qaeda http://www.lasexta.com/noticias/internacional/ liderqaeda-amenaza-repetir-mil-veces-atentados11s_2016091057d3e0e40cf29063b1bb7301.html

Prensa Latina | $\frac{\text { internet@granma.cu, 29 de }}{2016}$ 22:08:47,
agosto de
http://www.granma.cu/mundo/2016-08-29/america-
latina-pide-a-eeuu-revisar-politica-migratoria-hacia-
cuba-29-08-2016-22-08-47

Revista Forbes México, reporte Financiero, Pronóstico de Crecimiento Económico, septiembre 2016.

Sampieri, Roberto Hernández Carlos Fernández-Collado, Pilar Baptista Lucio, Metodología de la Investigación, Cuarta edición, pag. 111. 